\title{
ARBORICULTURA Y SILVOPASTORALISMO EN EL PERÍODO FORMATIVO (1.400 a.C.-500 d.C.) DE LA CUENCA DEL SALAR DE ATACAMA*
}

\author{
ARBORICULTURE AND SILVOPASTORALISM DURING THE FORMATIVE \\ PERIOD (1,400 BC-500 AD) OF THE SALAR DE ATACAMA BASIN
}

\author{
Virginia McRostie B. ${ }^{1}$
}

\begin{abstract}
Diversas hipótesis y modelos se han planteado en torno al papel que jugó la agricultura, horticultura, recolección, caza y ganadería de camélidos durante el periodo Formativo (1.400 a.C.-500 d.C.) en los oasis del Salar de Atacama y el Loa Medio. En este artículo se reevalúan estos planteamientos a la luz de nuevos datos obtenidos principalmente del análisis de microfósiles adheridos en palas líticas e instrumentos de molienda y en menor medida desde distintos isótopos (d13Ccol, d13Cap, d15N, d18O, $\mathrm{Sr}^{87} / \mathrm{Sr}^{86}$ ). Los datos se interpretan desde una perspectiva teórica que enfatiza el riesgo y una evolución no lineal en la interacción sociedadplanta. Se discute la funcionalidad de los instrumentos analizados y el manejo de recursos silvestres como Cactáceas y Ciperáceas además de tubérculos aún no identificados. Desde la arqueobotánica se sugiere que los bosques de algarrobo (Prosopis) no son nativos del área, sino que fueron producto de una arboricultura formativa posiblemente ligada a una economía silvopastoralista en los oasis. Ambas propuestas replantean la historia cultural y ecológica del área, enriquecen la discusión del Formativo, y la biogeografía de Prosopis en América.
\end{abstract}

Palabras claves: microfósiles, isótopos, arboricultura, silvopastoralismo, interacción sociedad-planta, Formativo.

Several hypotheses and models have been put forward about the role played by agriculture, horticulture, gathering, hunting and herding of camels during the Formative period (1,400 BC-500 AD) in the oases of the Salar de Atacama and the Middle Loa. This article re-assessed these proposals in light of new data obtained mainly from the analysis of microfossils attached to lithic hoes, grinding instruments and, to a lesser extent, from different isotopes (d13Ccol, d13Cap, d15N,d18O, $\left.\mathrm{Sr}^{87} / \mathrm{Sr}^{86}\right)$. The data is interpreted from a theoretical perspective that emphasizes risk and nonlinear evolution in human-plant interaction. I discuss the functionality of the instruments analyzed and the management of wild resources such as cacti and sedges, as well as unidentified tubers. From an archaeobotanical perspective I suggest that algarrobo (Prosopis) forests are not native to the area, but were the product of a formative arboriculture possibly linked to a silvopastoralist economy in the oases. Both proposals reconsider the cultural and ecological history of the area, and enrich the discussion of the Formative and of the biogeography of Prosopis in the Americas.

Key words: Microfossils, isotopes, arboriculture, silvopastoralism, human-plant interaction, Formative.

\section{Hipótesis sobre la Producción de Alimentos en el Salar de Atacama y el Loa Medio durante el Período Formativo}

En la Puna Occidental de Atacama la mayoría de los investigadores están de acuerdo en que la domesticación de camélidos y la ganadería precedió a la agricultura y que esta última vendría a complementar las prácticas pastoralistas más tardíamente (Agüero 2005; Agüero et al. 2009; Benavente 1988-1989; Núñez et al. 2009). Así la domesticación de camélidos por parte de las comunidades arcaicas de la fase Puripica-Tulán (3.000-1.800 a.C.), implicó "un nuevo modo de vida que trajo complejidad y mayores éxitos en términos de ajustes adaptativos antes de la implantación agrícola, creando las condiciones para el surgimiento del particular proceso de Neolitización de los Andes del Centro Sur" (Núñez y Santoro 2011:495). No obstante, mientras la domesticación de camélidos ha sido fundamentada mediante diferentes líneas de evidencia (Cartajena et al. 2007; Hesse 1982; Jackson y Benavente 2010; Núñez 1986-1987); el desarrollo agrícola ha sido discutido para el Formativo principalmente desde la presencia y/o ausencia de escasos macrorrestos de cultivos (Tabla 1), la aparición de palas líticas, la colonización de los oasis en el Salar de Atacama, mayores niveles de sedentarismo y complejidad social. Estos cambios se empiezan a percibir recién hacia el Formativo

$\overline{1}$ Cerro la Paloma 2 \#10915, Lo Barnechea, Santiago, Chile. virginia.mcrostie@gmail.com 
Tabla 1. Macrorrestos de cultivos. Crops macro remains.

\begin{tabular}{|c|c|c|c|c|}
\hline Período & Sitio & Taxa & Resto arqueobotánico & Referencia \\
\hline $\begin{array}{l}\text { Arcaico } \\
\text { Tardío }\end{array}$ & Tulan 52 & Lagenaria sp. & cáscara & McRostie 2007 \\
\hline $\begin{array}{l}\text { Formativo } \\
\text { Temprano }\end{array}$ & $\begin{array}{l}\text { Chiu Chiu } 200 \\
\text { Chiu Chiu } 200 \\
\text { Chiu Chiu } 200 \\
\text { Tulan } 109 \\
\text { Tulan 54 } \\
\text { Tulan 54, 55 } \\
\text { Tulan 55 } \\
\text { Tulan } 85\end{array}$ & $\begin{array}{l}\text { Gossypium sp. } \\
\text { Lagenaria sp. } \\
\text { Zea mays } \\
\text { Gossypium sp. } \\
\text { Cf. Chenopodium sp. } \\
\text { Lagenaria sp. } \\
\text { Zea mays } \\
\text { Zea mays }\end{array}$ & $\begin{array}{l}\text { fibra } \\
\text { cáscara } \\
\text { coronta }(n=3) \\
\text { fibra } \\
\text { semilla } \\
\text { cáscara } \\
\text { hojas, granos }(n=?) \\
\text { coronta }(n=2)\end{array}$ & $\begin{array}{l}\text { Benavente 1988-89 } \\
\text { Benavente 1988-89 } \\
\text { Benavente 1988-89 } \\
\text { Núñez et al. } 2006 \\
\text { McRostie } 2007 \\
\text { McRostie } 2007 \\
\text { Núñez et al. } 2006 \\
\text { Núñez et al. } 2006\end{array}$ \\
\hline $\begin{array}{l}\text { Formativo } \\
\text { Medio/Tardío }\end{array}$ & $\begin{array}{l}\text { Gatchi, Calar, Sequitor, } \\
\text { Tchaputchayna, Toconao O. } \\
\text { Puripica } 31 \\
\text { Ranl } 100 \\
\text { Ranl } 100 \\
\text { Topater } \\
\text { Topater } \\
\text { Tulan } 82 \\
\text { Tulan } 58 \\
\text { Tulor } 1 \\
\text { Tulor } 1\end{array}$ & $\begin{array}{l}\text { Lagenaria sp. } \\
\text { Zea mays } \\
\text { Lagenaria sp. } \\
\text { Zea mays } \\
\text { Erythroxylon coca } \\
\text { Phaseolus sp. } \\
\text { Zea mays } \\
\text { Capsicum sp. } \\
\text { Lagenaria sp. } \\
\text { Zea mays }\end{array}$ & $\begin{array}{l}\text { cáscara } \\
\text { macro }(n=?) \\
\text { cáscara } \\
\text { coronta }(n=>17) \\
\text { macro } \\
\text { semilla } \\
\text { coronta }(n=?) \\
\text { semilla } \\
\text { cáscara } \\
\text { coronta }(n=<10)\end{array}$ & $\begin{array}{l}\text { Vidal } 2007 \\
\text { Núñez } 2005 \\
\text { Pollard } 1971 \\
\text { Pollard } 1971 \\
\text { Thomas et al. } 1995 \\
\text { Thomas et al. } 1995 \\
\text { Núñez } 2005 \\
\text { Holden } 1991 \\
\text { Llagostera et al. 1984; Baron 1986 } \\
\text { Llagostera et al. 1984; Baron 1986; } \\
\text { Vidal 2007 }\end{array}$ \\
\hline Formativo & $\begin{array}{l}\text { Chorrillos } \\
\text { Ghatchi, Poconche } 12 \\
\text { Tulan 57, 59, 71, 82, } 85\end{array}$ & $\begin{array}{l}\text { Chenopodium sp. } \\
\text { Chenopodium sp. } \\
\text { Lagenaria sp. }\end{array}$ & $\begin{array}{l}\text { semilla } \\
\text { semilla } \\
\text { semilla, cáscara }\end{array}$ & $\begin{array}{l}\text { Belmar y Quiroz } 2005 \\
\text { Vidal } 2007 \\
\text { Núñez } 2005 \text {; Núñez et al. 2006; } \\
\text { Vidal } 2007\end{array}$ \\
\hline
\end{tabular}

Medio y/o Tardío (100 a.C. a 600 d.C.), ya que durante el Formativo Temprano o fase Tilocalar (1.400 a.C. a 400 d.C.) habría una continuidad de las prácticas pastoralistas y cazadoras recolectoras en las quebradas (Agüero 2005; Núñez et al. 2009). Durante el Formativo Medio o la fase Toconao (400 a.C.-100 d.C.), Llagostera et al. (1984) y Barón (1986) propusieron que Tulor aldea sería la primera aldea sedentaria y agropastoralista en los oasis del Salar de Atacama. Tarragó (1989) planteó que la complejidad social evidenciada en los contextos fúnebres de Toconao Oriente durante las fases Toconao y Séquitor (400 a.C.-500 d.C.) sería posible únicamente con una producción agraria que permitiera la generación excedentaria. Núñez (2005) propone que la colonización de los oasis de San Pedro después del Formativo Temprano (400 a.C.-600 d.C.) se realizó por parte de las sociedades ganaderas de las quebradas que bajan a los oasis buscando más tierras y mejores condiciones para soportar una complejidad creciente. Para él los asentamientos más estables en los oasis se relacionan con actividades agrícolas y a un poder sociopolítico representado por estas cabeceras agrarias que complementarían el recurso ganadero con poblaciones satélites en las quebradas. Mientras, Agüero (2005) y colaboradores (Agüero y Uribe 2011; Agüero et al. 2009; Vidal 2007) revisan y recuperan nuevas evidencias arqueobotánicas para los oasis y quebradas del margen norte del Salar de Atacama, enfatizando el recurso forestal Prosopis y su recolección como explicación de la colonización de los oasis que empezaría paulatinamente durante el Arcaico Tardío. A su vez proponen junto con Adán y Urbina (2007) una posible diferenciación entre las poblaciones quebradeñas que persisten en una subsistencia animal versus una población de oasis relacionada con la recolección forestal y horticultura de maíz especialmente hacia finales del Formativo. En el Loa Medio se ha planteado para 
el Formativo Temprano una población cazadora/ pastora con crecientes niveles de sedentarismo (Benavente 1982, 1988-1989; Pollard 1970, 1971) y una agricultura de maíz hacia la fase Loa II (100300 d.C.) cuando se consolida la vida sedentaria (Pollard 1971). También la evidencia de cerámica y arquitectura foránea llevaron a sugerir el arribo de poblaciones y/o la difusión de prácticas agrícolas desde otras áreas de la Circumpuna (Barón 1986; Benavente 1988-1989; Tarragó 1989).

Por tanto, todos estos modelos aceptan que la ganadería precedió a la agricultura y que posteriormente la explotación ya sea de Prosopis o el cultivo del maíz habría consolidado los distintos cambios formativos; si bien difieren en el rol que le otorgan a la intensidad de la producción agrícola, su tiempo de consolidación y su forma de complementación con la ganadería.

Estas diversas perspectivas sobre el rol de las plantas domésticas y silvestres son problemáticas no solo porque ofrecen un panorama parcial de las evidencias, sino también por una postura teórica que asume el maíz como un cereal equivalente a lo que fue el trigo y la cebada en el Neolítico del Viejo Mundo, asimilándose como diagnóstico y propulsor de un estilo de vida Formativo (Staller 2006). De esta misma manera, estos modelos han sugerido que la conformación de aldeas sedentarias, el incremento demográfico o la complejidad social estarían relacionados con el desarrollo agrícola. No obstante, en el área esta analogía con el Viejo Mundo (Bender 1978; Binford 1968; Childe 1951; Cohen 1977; Flannery 1969; Hayden 1995; H. E. Wright 1993) carece de sustento, ya que la mayoría de los sitios arcaicos y formativos tempranos no son ocupaciones densas en espacio o profundidad y no hay clara evidencia de fuerte y extensa jerarquización social (Agüero 2005; Aldunate et al. 1986; Benavente 1982; Druss 1976; Núñez et al. 2006; Núñez y Santoro 1988; Pollard 1971). Por otro lado, los proxies ambientales son ambiguos (p.ej., Betancourt et al. 2000; Geyh et al. 1999; Grosjean et al. 2003; Grosjean et al. 1997; Messerli et al. 1993; Rech et al. 2002; Valero-Garcés et al.1996); por tanto no deberían ser relacionados con cambios en las prácticas productivas, especialmente sin evidencia empírica que lo avale. De todas formas, si efectivamente hubiesen existido presiones sobre la producción alimentaria esta pudiera haber sido manejada de diversas maneras "el estrés alimentario no está necesariamente correlacionado con los inicios de la producción de alimentos" (Price y Gebauer 1995:4 [traducido por el autor]).

De esta manera, para llegar a un entendimiento más elaborado de la transición a la producción de alimentos vegetales se hacía necesario, por una parte, complementar el cuerpo de macrorrestos con otras evidencias y, por otra, plantear nuevas aproximaciones teóricas susceptibles de iluminar la diversidad y particularidad de este proceso en el área.

\section{El Riesgo en los Andes y Posturas Evolucionistas en la Interacción Sociedad-Planta}

Debido a la geografía y el ambiente extremo de los Andes del Sur el manejo del riesgo es considerado una de las principales estrategias económicas (Aldenderfer 2006; Browman 1987a, 1987b; Brush 1982; Custred 1977; Escola 1996; Goland 1993; Hesse 1982; Kuznar 2001; Olivera 1998; Winterhalder y Kennet 2006). Browman (1987b) ha sintetizado estas estrategias en (a) la generación de redes sociales (b) un patrón de asentamiento especializado (c) la diversificación de actividades productivas (d) el desarrollo de técnicas productivas para maximizar la explotación y el almacenamiento de recursos, y (e) incentivar la capacidad de carga para reducir el riesgo productivo. En la Puna de Atacama se han documentado varias de estas estrategias a través del tiempo (p.ej., Castro et al. 1984; Hidalgo 1985; Martínez 1998; Núñez y Dillehay 1979), y por tanto estas podrían haberse implementado para hacer frente a los distintos escenarios de la transición formativa.

A su vez, para entender los cambios productivos durante este período es necesario considerar el vasto terreno intermedio entre las prácticas recolectoras y agrícolas, evitando reducir el manejo de plantas a una dicotomía recolector o agricultor. Los modelos evolucionistas (Ford 1985; Harris 1989, 1996; Smith 2001) defienden la idea de una gradiente continua no unilineal desde la explotación de recursos silvestres a la agricultura intensiva y proveen una fructífera perspectiva para explorar la interacción sociedad-planta en las sociedades formativas de la Puna de Atacama.

Ford (1985) propone diferentes etapas y métodos de producción de alimentos. La primera etapa es la procuración de alimentos y se refiere exclusivamente a la utilización de plantas silvestres sin ningún tipo de intervención humana; la segunda etapa se refiere a la producción de alimentos y se divide en 
dos subetapas: cultivo y domesticación de especies silvestres y cultivo de plantas domesticadas.

Harris (1989) propone una continua y creciente interacción entre personas y plantas que finalmente lleva a la agricultura. Distingue cuatro etapas generales: (1) el procuramiento de plantas y animales silvestres (caza y recolección verdadera), (2) la producción de alimentos de plantas silvestres (los inicios del cultivo), (3) cultivo sistemático (de plantas morfológicamente silvestres) y, finalmente, (4) la agricultura basada en plantas domesticadas (Fuller 2007). Cada una de estas etapas refleja una intensificación de la producción que puede resultar en mayores niveles de sedentarismo, densidad poblacional y complejidad social. Sin embargo, este proceso no es unidireccional ni determinista y se compone de diferentes actividades y sistemas de producción de alimentos.

Smith (2001) define sociedades con "bajo nivel de producción de alimentos", en un punto medio entre la caza, la pesca, la recolección y la agricultura. Estas sociedades pueden reproducir o no reproducir plantas domésticas. Las primeras se caracterizan porque los cultivos contribuyen menos de $30-50 \%$ de las calorías totales anuales, mientras que las segundas cultivan solo plantas no domesticadas. Ambos modifican el medioambiente para mejorar la productividad de las especies seleccionadas.

"Las sociedades intermedias no son pálidos reflejos o extensiones lógicas de agricultores o cazadores-recolectores, sino una clase independiente de soluciones socioeconómicas a largo plazo, extremadamente variables y exitosas, ajustadas a una amplia gama de contextos culturales y ambientales locales" (Smith 2001:33-34 [traducido por el autor]).

El concepto de domesticación tiene diferentes implicancias. Para Smith (2001), las características centrales que definen la domesticación y la creación de recursos domésticos es la naturaleza de la relación entre recursos y humanos "la relación de la intervención iniciada es la domesticación" (Smith 2006:17 [traducido por el autor]). Smith (2006) reconoce que el cambio morfológico cae en el extremo final de lo que puede ser caracterizado como un proceso de tres partes que en forma simplista se puede resumir como cambio de comportamiento / cambios genéticos / cambios morfológicos (Smith 2006). Por lo tanto, diferentes marcadores pueden ser más eficaces en la detección de las diferentes etapas de este proceso que operan a diferentes escalas no solo de ciertas especies en particular, sino también en todo el paisaje y la vida social. En este sentido, además de la domesticación de especies, la domesticación del paisaje se ha utilizado en modelos evolutivos para rastrear los orígenes de la agricultura y los procesos que conducen a la misma (Clement 1999; Harlan 1975; Rindos 1984; Terrell et al. 2003).

\section{Materiales y Metodología}

Artefactos que históricamente se han relacionado con labores agrícolas o al procesamiento vegetal (palas líticas e instrumentos de molienda) fueron muestreados para análisis múltiple de microfósiles (Babot 2004; Coil et al. 2003; Korstanje 2005).

Los artefactos muestreados provienen de sitios del período Arcaico Tardío y Formativo (Figura 1) disponibles únicamente en colecciones museográficas, si bien ciertos sitios presentaban material in situ (Tabla 2). Para identificar los microfósiles arqueológicos se confeccionó una colección de referencia de plantas silvestres y cultivadas además de considerar otras descripciones para plantas andinas y americanas (McRostie 2013).

Este análisis se complementó con una pequeña muestra de restos humanos para isótopos $(\mathrm{d} 13 \mathrm{Ccol}$, d13Cap, d15N, d18O, $\mathrm{Sr}^{87} / \mathrm{Sr}^{86}$ ) de modo de poder evaluar el consumo de plantas y posibles grupos interactuando. Se confeccionó además una pequeña referencia de plantas que junto con antecedentes previos permitió una interpretación preliminar de los valores arqueológicos (McRostie 2013).

\section{Resultados}

Las muestras analizadas provienen de 20 sitios correspondientes al Arcaico Tardío (Kalina, Ghatchi 2C, Puripica 1, Tulan 52); Formativo Temprano (Chiu Chiu 200, Tulan 54, 55, 122); Formativo Tardío (Ranl 273, Calar, Puripica 23, 31; Tulor 1, Toconao, Tulan $57,58,82$ ) y Formativo en general (Ghatchi 2C (componente Formativo), Tulan 67, Tulan 85, Chorrillos). Los fitolitos se encontraron en 88 artefactos, los granos de almidón en 43, las diatomeas en 23 , el polen en 13 , las esferulitas en seis, los oxalatos en tres, mientras que 55 artefactos (36\%) no tenían microfósiles. Sin embargo, solo una parte de estos microfósiles fueron clasificados como culturales siguiendo una serie de variables tafonómicas, taxonómicas, ecológicas y arqueológicas (McRostie 2013). 


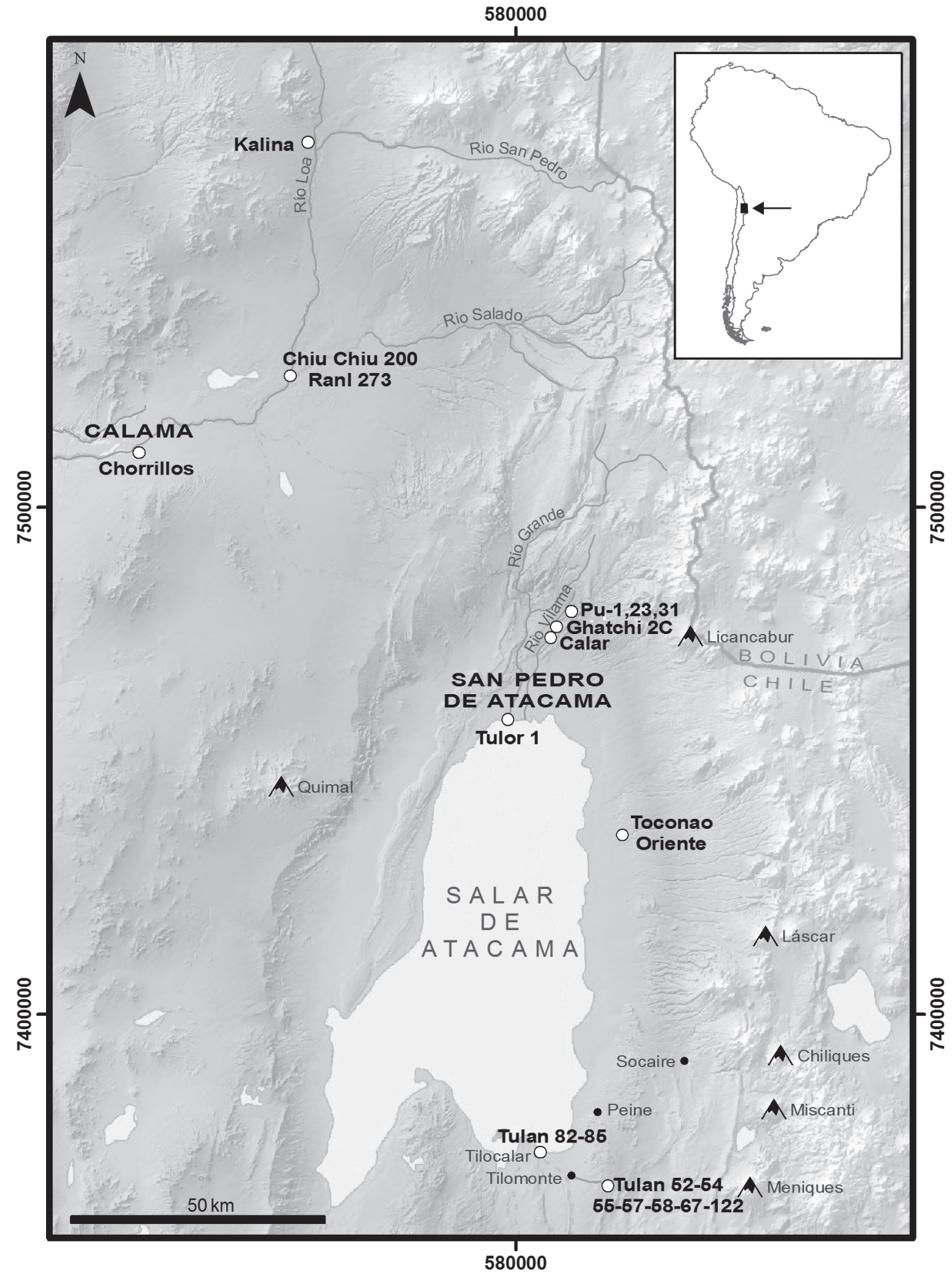

- Sitios Arqueológicos / Archaeological Sites

Figura 1. Mapa del área y sitios muestreados.

Map of the area and sites sampled. 
Tabla 2. Sitios y artefactos muestreados para análisis de microfósiles. Sites and artefacts sampled for microfossil analyses.

\begin{tabular}{|c|c|c|c|c|c|c|c|}
\hline Período & Subárea & Sitio & Mano & Mortero & Pala & Total & Proveniencia \\
\hline \multirow{3}{*}{$\begin{array}{l}\text { Arcaico } \\
\text { Tardío }\end{array}$} & Salar & Ghatchi $2 \mathrm{C}$ & 3 & 12 & - & 15 & in situ \\
\hline & Loa & Kalina & 2 & 10 & - & 12 & in situ \\
\hline & Salar & Puripica 1 & 1 & - & - & 1 & Museo G. Le Paige \\
\hline Total & - & - & 6 & 22 & - & 28 & - \\
\hline \multirow{4}{*}{$\begin{array}{l}\text { Formativo } \\
\text { Temprano }\end{array}$} & Salar & Tulan 55 & - & - & 1 & 1 & \multirow{4}{*}{$\begin{array}{l}\text { Museo G. Le Paige } \\
\text { Universidad de Chile } \\
\text { Museo G. Le Paige } \\
\text { in situ }\end{array}$} \\
\hline & Loa & Chiu Chiu 200 & 8 & 3 & 7 & 18 & \\
\hline & Salar & Tulan 54 & 3 & 5 & - & 8 & \\
\hline & Salar & Tulan 122 & - & 1 & - & 1 & \\
\hline Total & - & - & 11 & 9 & 8 & 28 & - \\
\hline \multirow{8}{*}{$\begin{array}{l}\text { Formativo } \\
\text { Medio/Tardío }\end{array}$} & Salar & Puripica 31 & 5 & - & 4 & 9 & Museo G. Le Paige \\
\hline & Salar & Puripica 23 & 2 & - & 5 & 7 & Museo G. Le Paige \\
\hline & Salar & Calar & 2 & - & 6 & 8 & Museo G. Le Paige \\
\hline & Loa & RanL 273 & 4 & 2 & 2 & 8 & Museo G. Le Paige \\
\hline & Salar & Tulan 57 & 3 & - & 4 & 7 & Museo G. Le Paige \\
\hline & Salar & Tulan 58 & 1 & - & - & 1 & Museo G. Le Paige \\
\hline & Salar & Tulor 1 & 13 & - & 9 & 22 & Museo G. Le Paige \\
\hline & Salar & Tulan 82 & 1 & - & - & 1 & Museo G. Le Paige \\
\hline Total & - & - & 31 & 2 & 30 & 63 & - \\
\hline \multirow{4}{*}{ Formativo } & Salar & Ghatchi $2 \mathrm{C}$ & 2 & 5 & - & 7 & in situ \\
\hline & Salar & Tulan 67 & - & - & 1 & 1 & Museo G. Le Paige \\
\hline & Salar & Tulan 85 & 1 & 9 & - & 10 & Museo G. Le Paige \\
\hline & Loa & Chorrillos & - & - & 13 & 13 & Museo El Loa \\
\hline Total & - & - & 3 & 14 & 14 & 31 & - \\
\hline Total general & - & - & 51 & 47 & 52 & 150 & - \\
\hline
\end{tabular}

Los artefactos con microfósiles culturales son 43 y representan un $28 \%$ del universo muestreado. Dentro de estos tipos "culturales" se identificaron 10 plantas. Seis fueron identificadas exclusivamente por granos de almidón: cf. Capsicum, cf. Cucurbitaceae, cf. Geoffroea decorticans, cf. Hoffmannseggia sp., cf. tubérculos y cf. Zea/Prosopis, mientras dos plantas fueron identificadas exclusivamente con fitolitos: cf. Opuntia sp. y cf. Phragmites. Una especie se identificó con almidón y fitolitos: cf. Scirpus, y una por oxalato y almidón: cf. Prosopis. Las esferulitas de camélidos también se discuten como datos culturales. Se refuerza la similitud entre los granos de almidón regulares de Prosopis y Zea mays (Giovannetti et al. 2008; Lema et al. 2012) que por ahora no permite excluir uno del otro.

De estas 10 plantas identificadas, solo un $20 \%$ se reconoció como doméstico (cultivos), un $20 \%$

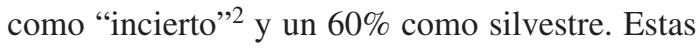

plantas nos permiten visualizar por primera vez qué recursos se han manipulado y procesado con los artefactos analizados en los distintos sitios (Tabla 3).

\section{Interpretación y Discusión}

Corroborando antecedentes arqueobotánicos previos (McRostie 2007; Núñez et al. 2009; Vidal 2007), las plantas silvestres fueron predominantes durante el Arcaico Tardío así como en el período Formativo. Recursos como cf. Scirpus, cf. Hoffmannseggia (tubérculo) y cf. Opuntia están presentes en ambos períodos, sin embargo, existe una diversificación para el período Formativo con la introducción y preponderancia de cf. Prosopis, más tubérculos, cf. Geoffroea y una baja presencia de Capsicum; Cucurbitaceae y Zea mays, el cual parece no haber tenido un impacto importante en las economías formativas de los Andes del Sur 
Tabla 3. Taxa presente en artefactos por sitios y períodos (McRostie 2013).

Taxa present on artefacts through sites and periods (McRostie 2013).

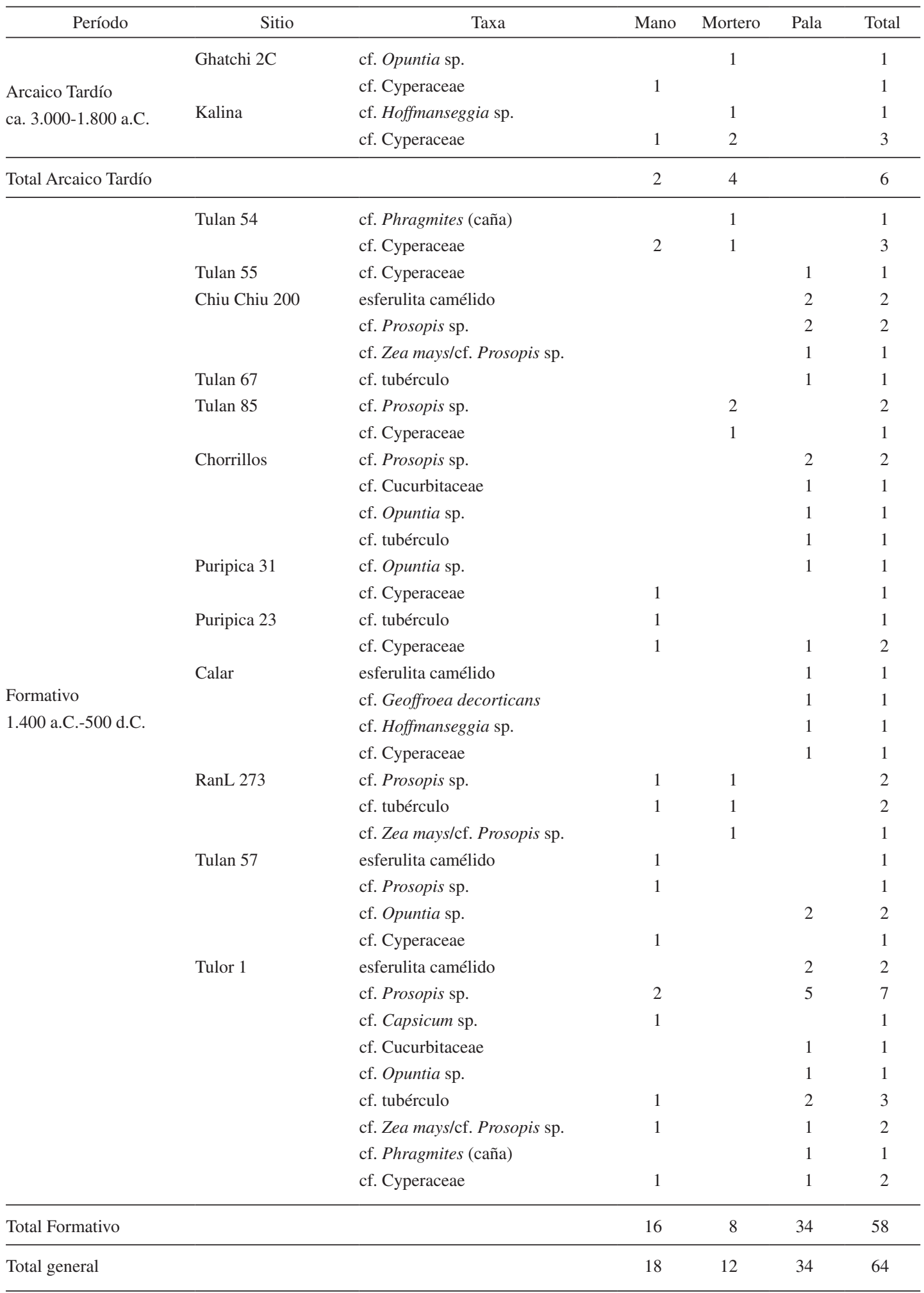


hasta solo después de algunos milenios (Drennan 1996; Logan et al. 2012; Minnis 1992; Olivera y Yacobaccio 1999; Pearsall 2007; Rivera 2005; Smith 2001; Staller y Thompson 2000). En este sentido, el consumo de maíz no es fácil de interpretar debido a los distintos modelos para el fraccionamiento de la apatita (Tykot et al. 2009), sin embargo C3 representaría más del $50 \%$ en la dieta total. Las valores altos de d13Cap podrían corresponder a plantas C4 o CAM (Opuntia, Echinopsis) (McRostie 2013) las que están bien representadas en el registro arqueobotánico (Holden 1991; McRostie 2007; Núñez et al. 2009; Vidal 2007). Si bien el maíz también podría estar incidiendo en estos valores (Tabla 4), pareciera que durante el período Formativo las plantas $\mathrm{C} 4$ no eran un alimento básico como durante el período Intermedio Tardío (1.100-1.400 d. C.), cuando un valor d13Cap $=-6,7$ está presente en Caspana (Knudson y Torres-Rouff 2009). En cuanto al porcentaje de C4 en la proteína, $40 \%$ de los individuos muestran la presencia de $\mathrm{C} 4$ entre el $10 \%$ y el $70 \%$. Esto podría ser debido a la dieta enriquecida $\mathrm{C} 4$ de los camélidos que se consumen como carne y el efecto carnívoro del amamantamiento en algunos neonatos. Los valores de nitrógeno son difíciles de relacionar con la dieta, debido a la fuerte influencia de la aridez (Bustamante et al. 2004; Coltrain et al. 2006). Los isotopos de $\mathrm{Sr}$ y d18O no permiten proponer el arribo de poblaciones o poblaciones internamente diferenciadas por ahora. Solo un individuo adulto de Tulan 58 (C7) tiene valores radiogénicos equiparables a la fauna del área de Tiwanaku (Knudson 2008) (Tabla 4).

Por tanto, este Formativo se basa principalmente en el manejo de plantas locales, las que en contextos arqueológicos de todo el mundo han sido reportadas como relevantes en la dieta (Babot 2004; Beresford-Jones 2011; Casas et al. 1997; Danielson y Reinhard 1998; Flannery 1968; Holden 1991; Messner 2008; Minnis 1992; Pearsall 1989; Whitehead 2006; Wollstonecroft et al. 2008). En este sentido el manejo y cultivo prolongado de estas especies probablemente las llevó a diferentes etapas del proceso de domesticación que requieren ser exploradas a futuro desde una perspectiva interdisciplinaria.

La correlación entre palas y agricultura debe ser reevaluada. Esta herramienta como se indica por varios autores (Haber y Gastaldi 2006; Núñez 1992; Núñez et al. 2003; Pérez 2010; Tamblay
1990; Uribe y Carrasco 1999; Whitehead 2006), podría ser útil para múltiples propósitos como para minería, la extracción de Ciperáceas y tubérculos, la excavación de pozos y construcción de casas o manipulación de guano ya sea para fertilizante o combustible. En este sentido podrían estar en estrecha relación con el patrón más sedentario observado en Atacama. Tal como señala Kelly (1992) la reducción de la movilidad implica un cambio en la gestión de los residuos. Así, en Atacama y durante el período Formativo, estas herramientas podrían estar relacionadas con las actividades de intensificación de plantas, pero también a múltiples tareas relacionadas con el manejo del suelo, del agua y la construcción de una forma más estable de vida.

Los patrones de plantas inferidos desde los artefactos de molienda no muestran exclusión entre las formas cónicas y planas características del Arcaico y el Formativo respectivamente (Tabla 3), sugiriendo que no hay una relación entre el cambio en la forma y los recursos procesados (Adams 1993, 1999; Liu et al. 2011; Wright 1994). Los morteros planos han sido reportados por varios autores como más eficientes que los cónicos, en cuanto implican menos esfuerzo y tiempo de trabajo para la misma cantidad de producto elaborado (Adams 1993; Babot 2006; Mauldin 1993; Wright 1994). Además el recurso molido tendría una mayor bioaccesibilidad (Babot 2004; Stahl 1989; Wollstonecroft et al. 2008; K. Wright 1993, 1994), si bien también podría relacionarse con nuevas recetas adecuadas para el fortalecimiento de las redes sociales o el incremento de demandas sociales evidenciadas hacia fines del período Formativo (Agüero y Uribe 2011).

\section{Un Nuevo Modelo para el Formativo de la Puna de Atacama Occidental}

Al complementar los antecedentes arqueobotánicos disponibles hasta la fecha, se reafirma que durante el Formativo hay una continuidad en el cultivo de plantas silvestres. La falta de fitolitos de maíz en las palas impide proponer su cultivo al menos en grandes cantidades (Logan et al. 2012; McRostie 2013), y la incierta identificación de los tubérculos merece futuros análisis para establecer mejor sus implicancias productivas. Sin embargo, me parece que la reiterada ausencia de Prosopis (a nivel de macro y microrresto) durante el Arcaico y su aparición e incremento durante el Formativo (Druss 


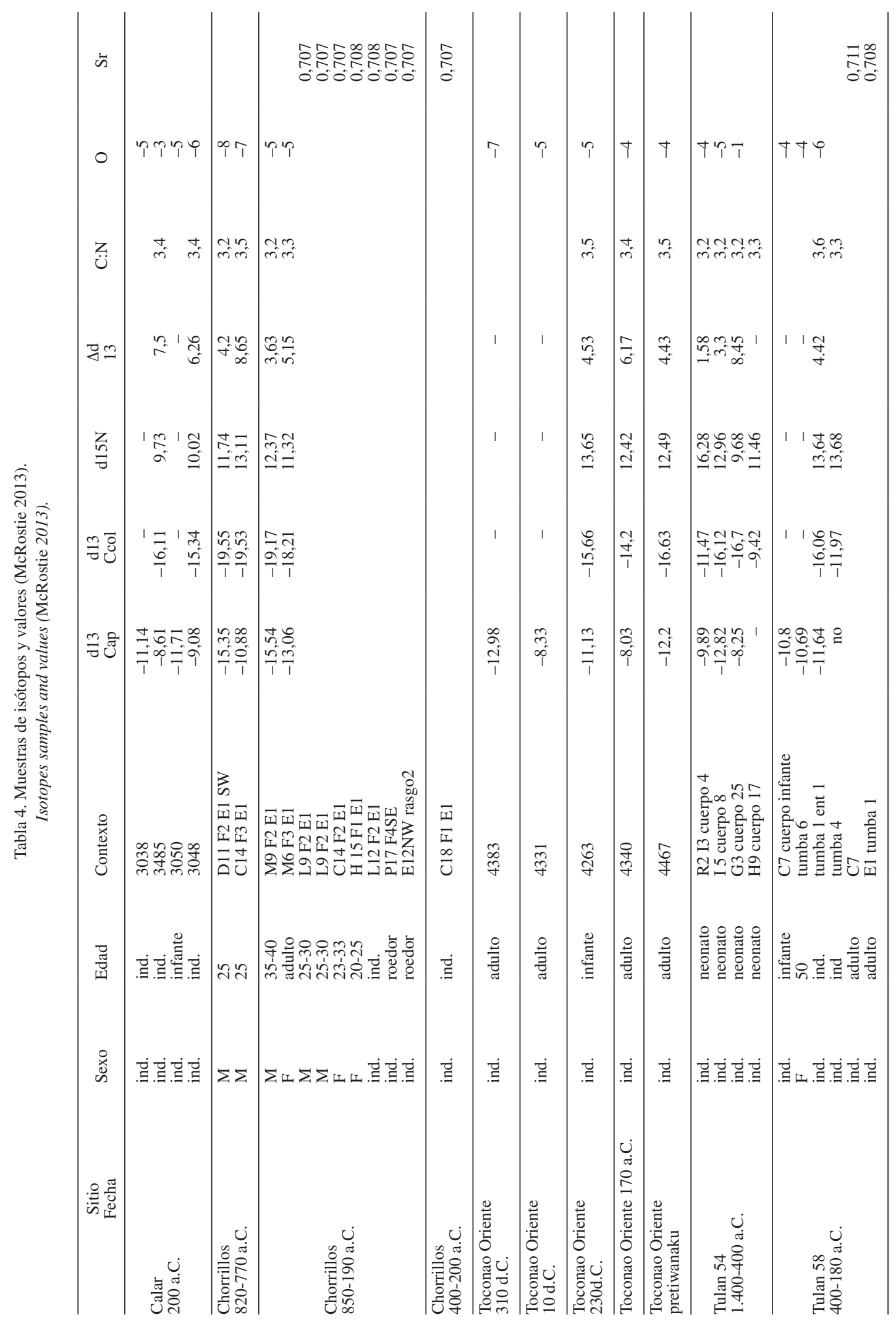


1976; Holden 1991; McRostie 2007, 2013; Vidal 2007) requiere una reevaluación más detallada ${ }^{3}$.

En general la biogeografía de Prosopis aun es fragmentaria para América, no obstante es durante el Cuaternario Tardío que se infieren procesos de dispersión (Bessega et al. 2006). En la zona de estudio los proxies paleoambientales no proporcionan evidencia de la existencia de los bosques de Prosopis; si bien en la Pampa del Tamarugal $\left(21^{\circ} \mathrm{S}\right)$ Nester et al. (2007) encuentra Prosopis alrededor de 16.380 a 13.740 a.p. No obstante, este podría ser Prosopis tamarugo, especie nativa del área, mientras Prosopis alba podría estar más relacionado con la actividad humana (Gayó et al. 2012). En este sentido varios autores sugieren que esta última especie fue introducida a Chile desde Argentina en época precolombina (Ehleringer et al. 1992; Rodríguez et al. 1983). En los oasis de Atacama no está clara la taxonomía de Prosopis, pero al parecer $P$. alba sería la especie predominante (Víctor Quezada comunicación personal 2013), y si bien aún falta esclarecer muchas variables para determinar el origen y/o vías de ingreso de estos árboles, Palacios y Brizuela señalan que

Los bosques de algarrobo de San Pedro de Atacama, descritos por Oviedo y Valdés (1535), merecen una mención especial porque son producto de la actividad de los agricultores que han habitado los oasis de San Pedro. No está claro de dónde vinieron las semillas fundacionales, los análisis exo-morfológicos sugieren similitudes con individuos de Prosopis alba de algunas localidades de Salta (Argentina) (Palacios y Brizuela 2005:42).

En Prosopis las plantas silvestres y cultivadas coexisten y numerosos programas forestales han demostrado que después de un primer cultivo estos bosques se expanden y naturalizan (Galera 2000). En Atacama no hay información etnobotánica en cuanto al manejo silvícola de Prosopis, no obstante, Casas y Caballero (1996) dan algunos conceptos para un árbol similar (Leucaena esculenta, guaje, Fabaceae) en la región mixteca de Guerrero, México. Ellos reconocen tres formas de manejo: cultivo, recolección de poblaciones silvestres, y retención selectiva o la tolerancia de individuos particulares. El cultivo consiste en la siembra de variedades seleccionadas en jardines o campos agrícolas. La tolerancia consiste en seleccionar guajes en función de ciertas propiedades (p. ej., dulce o amargo) y cortar los árboles indeseables. Asimismo la recolección de árboles silvestres es selectiva. De esta manera el manejo de estos árboles lleva a modificar la estructura fenotípica de las poblaciones de plantas.

Para Atacama, el cultivo de estos árboles podría haber comenzado en los albores del Formativo y después de una primera siembra, actividades como la tolerancia o la retención selectiva, entre otras, podrían haber tenido lugar. Por lo tanto, esta interacción sociedad-planta llevaría a modificar este género, así como el paisaje de los oasis haciendo su colonización efectiva y viable. "Ningún otro árbol del desierto tiene una influencia más penetrante sobre la vegetación vecina, suelos, microclima, la fauna y las poblaciones de insectos" Beresford-Jones 2011:214 [traducido por el autor]). En este punto, la naturaleza y el escenario de esta domesticación no puede ser plenamente definido, sin embargo, es muy probable que Prosopis se debería discutir como un cultígeno (Smith 2001) más que como un recurso silvestre y/o domesticado o semidomesticado como Martínez (1998) ya ha propuesto.

Por último, reconociendo la perspectiva de la diversificación en el manejo del riesgo, la implantación de los bosques no debe ser descrita como el principal motor para la ocupación de los oasis. Un sesgo en la interpretación de los cambios en los patrones de asentamiento del Formativo ha sido la dicotomización de animales y plantas en la economía (Agüero 2005; Núñez 2005). Así las prácticas de pastoreo en los oasis se han descartado por varias razones (Agüero 2005; Núñez 19861987, 1995), posiblemente reforzadas por las actividades actuales, que reflejan una larga historia de colonización y sustitución de las prácticas indígenas. Sin embargo, numerosos autores han documentado que los camélidos tienen una buena adaptación a altitudes bajas (Cadwallader et al. 2012; Finucane et al. 2006; Guillet 1987; Shimada y Shimada 1985; Wheeler 1995). A nivel local, Tulán 85 es un ejemplo de pastoreo en bajas altitudes, y en Tulor 1 existe una fuerte evidencia de la explotación y mantención de camélidos (Barón 1986; Llagostera et al. 1984).

Sugiero entonces que en el área del Salar de Atacama, las poblaciones del Formativo habían ganado el control de sus rebaños de camélidos y podían correr el riesgo de abandonar progresivamente las quebradas y ocupar los oasis, precisamente porque ahora podían moverse con su principal recurso -los 
camélidos-. En este sentido, las estrategias de riesgo permiten examinar el contexto en que la gente puede explorar nuevas vías de subsistencia (McClure et al. 2006). De esta manera, la colonización de los oasis por los pastores no significaba una amenaza para la supervivencia, ya que había una serie de estrategias en juego como una continuidad y diversificación en el uso del espacio y recursos (Minnis 1992), un desarrollo de las redes sociales para tener acceso directo o indirecto a distintos ecosistemas, además de nuevas tecnologías que les permitirían aumentar la capacidad de almacenamiento y/o la bioaccesibilidad entre otras.

Por tanto, si las sociedades formativas estaban manejando los bosques al mismo tiempo que sus rebaños en los oasis, entonces creo que una actividad "silvopastoralista" debería ser considerada como parte del sistema económico existente. "Este sistema alternativo de producción aprovecha la sinergia entre árboles y rebaño con efectos beneficiosos para el medio ambiente, la economía y la sociedad" (Ferreira De Mattos 2006:6). Los árboles de Prosopis son ideales en los sistemas silvopastorales de ambientes áridos (Galera 2000). Esta actividad a su vez habría favorecido la dispersión endozoocaria de Prosopis, la fertilización del suelo y en el tiempo talvez un sistema agrosilvopastoril. Harrison (1996) se refiere a la arboricultura como un sistema agrícola en España y Portugal durante la prehistoria. "En las discusiones de los sistemas agrícolas, los cultivos de árboles suelen recibir poca atención" (Harrison 1996:363 [traducido por el autor]). Describe las dehesas como comunidades distintivas de plantas manejadas por la sociedad, en donde los animales son usados para cosecharlas y convertirlas en guano, carne y trabajo. Desde el punto de vista de los pastores la dehesa entrega más beneficios que el cultivo de cereales, por su longevidad y refugio para los animales.

Una perspectiva silvopastoril y arborícola introduce nuevos elementos que no han sido discutidos antes y propone un caso especial para el período Formativo en las tierras áridas de los Andes del Sur. No obstante, los datos aún son escasos y muchos más análisis deben realizarse con el fin de probar y evaluar los diferentes aspectos e implicaciones de este modelo, como por ejemplo su coexistencia con otras formas productivas, su escala y/o temporalidad.

Agradecimientos: Entre muchos otros, a Conicyt Becas Chile, Dr. Jane Evans (NERC Isotope Geosciences Laboratory); Dr. Bill Sillar, Dr. Arlene Rosen; Mariana Ugarte por la elaboración del mapa y los evaluadores de este artículo.

\section{Referencias Citadas}

Adams, J.L. 1993. Toward understanding the technological development of manos and metates. Kiva 58:331-344.

----1999. Refocusing the role of food-grinding tools as correlates for subsistence strategies in the U.S. Southwest. American Antiquity 64:475-498.

Adán, L. y S. Urbina 2007. Arquitectura Formativa en San Pedro de Atacama. Estudios Atacameños 34:7-30.

Agüero, C. 2005. Aproximación al asentamiento humano temprano en los oasis de San Pedro de Atacama. Estudios Atacameños 30:29-60.

Agüero, C. y M. Uribe 2011. Las sociedades formativas de San Pedro de Atacama: Asentamiento, cronología y proceso. Estudios Atacameños 42:53-78.

Agüero, C., M. Uribe y C. Carrasco 2009. Current research in San Pedro de Atacama. Andean Past 9:323-328.

Aldenderfer, M. 2006. Costly signaling, the sexual division of labour, and animal domestication in the Andean Highlands. En Behavioral Ecology and the Transition to Agriculture, editado por D. Kennet y B. Winterhalder, pp. 167-196. University California Press, London.
Aldunate, C., J. Berenguer, V. Castro, L. Cornejo, J.L. Martínez y C. Sinclaire 1986. Sobre la cronología del Loa Superior. Chungara 16/17:333-346.

Babot, M.P. 2004. Tecnología y Utilización de Artefactos en el Noroeste Prehispánico. Tesis para optar al grado de Doctor en Arqueología, Facultad de Ciencias Naturales \& I.M.L., Universidad Nacional de Tucumán, Tucumán.

----2006. El papel de la molienda en la transición hacia la producción agropastoril: Un análisis desde la Puna Meridional Argentina. Estudios Atacameños 32:75-92.

Barón, A.M. 1986. Tulor: posibilidades y limitaciones de un ecosistema. Chungara 16/17:149-158.

Belmar, C. y L. Quiroz 2005. Informe de análisis carpológico. Cementerio Regimiento Chorrillos. Manuscrito inédito en posesión del autor.

Benavente, A. 1982. Chiuchiu 200: Una comunidad pastora temprana en la Provincia del Loa (II Región). Actas del IX Congreso Nacional de Arqueología Chilena, pp. 75-94. Sociedad Chilena de Arqueología, La Serena.

----1988-1989. Nuevas evidencias arqueológicas acerca de los asentamientos tempranos en el Loa Medio. Paleoetnológica 5:65-71. 
Bender, B. 1978. Gatherer-hunter to farmer: A social perspective. World Archaeology 10:204-222.

Beresford-Jones, D.G. 2011. The Lost Woodlands of Ancient Nazca. Oxford University Press, Oxford.

Bessega, C., J.C. Vilardi y B.O. Saidman 2006. Genetic relationships among American species of the genus Prosopis (Mimosoideae, Leguminosae) inferred from ITS sequences: Evidence for long-distance dispersal. Journal of Biogeography 33:1905-1915.

Betancourt, J., C. Latorre, J. Rech, J. Quade y K. Rylander 2000. A 22000-Year record of monsoonal precipitation from Northern Chile's Atacama Desert. Science 289:1542-1546.

Binford, L.R. 1968. Post-Pleistocene adaptations. En New Perspectives in Archaeology, editado por S. Binford y L. Binford, pp. 313-342. Aldine, Chicago.

Browman, D. 1987a. Agro-pastoral risk management in the Central Andes. Research in Economic Anthropology 8:171-200.

----1987b. Introduction: risk management in Andean arid lands. En Arid Land Use Strategies and Risk Management in the Andes. A Regional Anthropological Perspective, editado por D. Browman, pp. 1-23. Westview, Boulder.

Brush, S.B. 1982. The natural and human environment of the Central Andes. Mountain Research and Development 2:19-38.

Bustamante, M.M.C., L.A. Martinelli, D.A. Silva, P.B. Camargo, C.A. Klink, T.F. Domingues y R.V. Santos 2004. 15N natural abundance in woody plants and soils of Central Brazilian Savannas (Cerrado). Ecological Applications 14:200-213.

Cadwallader, L., D.G. Beresford-Jones, O. Whaley y T. O'Connell 2012. The signs of maize? A reconsideration of what $\delta 13 \mathrm{C}$ values say about palaeodiet in the Andean Region. Human Ecology 40:487-509.

Cartajena, I., L. Núñez y M. Grosjean 2007. Camelid domestication on the western slope of the Puna de Atacama, Northern Chile. Anthropozoologica 42:155-173.

Casas, A. y J. Caballero 1996. Traditional management and morphological variation in Leucaena esculenta (Fabaceae: Mimosoideae) in the Mixtec Region of Guerrero, Mexico. Economic Botany 50:167-181.

Casas, A., B. Pickersgill, J. Caballero y A. Valiente-Banuet 1997. Ethnobotany and domestication in Xoconochtli, Stenocereus stellatus (Cactaceae), in the Tehuacán Valley and La Mixteca Baja, México. Economic Botany 51:279-292.

Castro,V., C. Aldunate y J. Berenguer 1984. Orígenes altiplánicos de la Fase Toconce. Estudios Atacameños 7:159-178.

Childe, G.V. 1951. Man Makes Himself. New American Library, New York.

Clement, C. 1999. 1492 and the Loss of Amazonian Crop Genetic Resources. I. The Relation between domestication and human population decline. Economic Botany 53:188-202.

Cohen, M. 1977. Population pressure and the origins of agriculture. An archaeological example from the Coast of Peru. En Origins of Agriculture, editado por C.A. Reed, pp. 135-177. Mouton, The Hague.
Coil, J., M.A. Korstanje, S. Archer y C. Hastorf 2003. Laboratory goals and considerations for multiple microfossil extraction in archaeology. Journal of Archaeological Science 30:991-1008.

Coltrain, J.B., J.C. Janetski y C.W. Shawn 2006. The stable and radio-isotope chemistry of eastern Basketmaker and Pueblo groups in the four corners region of the American Southwest: implications for Anasazi diets, origins, and abandonments in Southwestern Colorado. En Histories of Maize: Multidisciplinary Approaches to the Prehistory, Linguistics, Biogeography, Domestication and Evolution of Maize, editado por J. Staller, R. Tykot y B.F. Benz, pp. 276-285. Elsevier, Amsterdam.

Custred, G. 1977. Las punas de los Andes Centrales. En Pastores de Puna: Uywamichiq Punarunakuna, editado por J. Flores Ochoa, pp. 55-86. Instituto de Estudios Peruanos, Lima.

Danielson, D.R. y K.J. Reinhard 1998. Human dental microwear caused by calcium oxalate phytoliths in prehistoric diet of the lower Pecos region, Texas. American Journal of Physical Anthropology 107:297-304.

Drennan, R. 1996. Betwixt and between in the Intermediate Area. Journal of Archaeological Research 4:95-131.

Druss, M. 1976. Medio ambiente, economía de subsistencia y patrones de asentamiento del Complejo Chiu Chiu (ca. 3000 a 2000 a.C), Norte de Chile. Estudios Atacameños 4:19-24.

Ehleringer, J.R., H.A. Mooney, P.W. Rundel, D.R. Evans, B. Palma y J. Delatorre 1992. Lack of nitrogen cycling in the Atacama Desert. Nature 359:316-318.

Escola, P. 1996. Riesgo e incertidumbre en economías agropastoriles: consideraciones teórico-metodológicas. Arqueología 6:9-24.

Ferreira De Mattos, G.A. 2006. Silvopastoral Systems in Latin America (22 April 2007). http://encyclopediaofforestry.org/index. php/Silvopastoral_Systems_in_Latin_America (14 Julio 2013).

Finucane, B., P.M. Agurto y W.H. Isbell 2006. Human and animal diet at Conchopata, Peru: stable isotope evidence for maize agriculture and animal management practices during the Middle Horizon. Journal of Archaeological Science 33:1766-1776.

Flannery, K.V. 1968. Archaeological systems theory and Early Mesoamerica. En Anthropological Archaeology in The Americas, editado por B. Meggers, pp. 67-87. Anthropological Society of Washington, Washington, D.C.

----1969. Origins and the ecological effects of early domestication in Iran and the Near East. En The Domestication and Exploitation of Plants And Animals, editado por P.J. Ucko y G.W. Dimbleby, pp. 73-100. Duckworth, London.

Ford, R.I. 1985. The processes of plant food production in prehistoric North America. En Prehistoric Food Production in North America, editado por R.I. Ford, pp. 1-18. Museum of Anthropology, University of Michigan, Ann Arbor.

Fuller, D. 2007. Contrasting patterns in crop domestication and domestication rates: Recent archaeobotanical insights from the Old World. Annals of Botany 100:903-924.

Galera, F.M. 2000. Los Algarrobos. Las Especies del Género Prosopis (Algarrobos) de América Latina con Especial Énfasis en Aquellas de Interés Económico. UNC-Secretaría de Ciencia y Tecnología; FAO, Córdoba. 
Gayó, E.M., C. Latorre, T.E. Jordan, P.L. Nester, S.A. Estay, K.F. Ojeda y C.M. Santoro 2012. Late Quaternary hydrological and ecological changes in the hyperarid core of the northern Atacama Desert (21으. Earth-Science Reviews 113:120-140.

Geyh, M.A., M. Grosjean, L. Núñez y U. Schotterer 1999. Radiocarbon Reservoir Effect and the Timing of the Late-Glacial/ Early Holocene Humid Phase in the Atacama Desert (Northern Chile). Quaternary Research 52(2):143-153.

Giovannetti, M.A., V.S. Lema, C.G. Bartoli y A.M. Capparelli. 2008. Starch grain characterization of Prosopis chilensis (Mol.) Stuntz and P. flexuosa DC, and the analysis of their archaeological remains in Andean South America. Journal of Archaeological Science 35:2973-2985.

Goland, C. 1993. Field scattering as agricultural risk management: A case study from Cuyo, Department of Puno, Perú. Mountain Research and Development 13:317-338.

Grosjean, M., I. Cartajena, M.A. Geyh y L. Nuñez 2003. From proxy data to paleoclimate interpretation: the mid-Holocene paradox of the Atacama Desert, northern Chile. Palaeogeography, Palaeoclimatology, Palaeoecology 194:247-258.

Grosjean, M., L. Núñez, I. Cartajena y B. Messerli 1997. MidHolocene climate and culture change in the Atacama Desert, northern Chile. Quaternary Research 48:239-246.

Guillet, D. 1987. On the potential for intensification of agropastoralism in the arid zones of the central Andes. En Arid Land Use Strategies and Risk Management in the Andes a Regional Anthropological Perspective, editado por D. Browman, pp. 81-98. Westview Press, Boulder.

Haber, A.F. y M.R. Gastaldi 2006. Vida con palas. Antípoda 2:275-302.

Harlan, J.R. 1975. Crops And Man. American Society of Agronomy, Madison.

Harris, D. 1989. An evolutionary continuum of people-plant interaction. En Foraging and Farming: The Evolution of Plant Exploitation, editado por D.R. Harris y G.C. Hillman, pp. 11-24. Unwin Hyman, London.

----1996. Introduction: themes and concepts in the study of early agriculture. En The Origins and Spread of Agriculture and Pastoralism in Eurasia, editado por D. Harris, pp. 1-11. Smithsonian Institution Press, Washington, DC.

Harrison, R.J. 1996. Aboriculture in Southwest Europe: Dehesas as managed woodlands. En The Origins and Spread of Agriculture and Pastoralism in Eurasia, editado por D. Harris, pp. 363-369. Smithsonian Institution Press, Washington DC.

Hayden, B. 1995. A new overview of domestication. En Last Hunters, First Farmers: New Perspectives on the Prehistoric Transition to Agriculture, editado por T.D. Price y A.B. Gebauer, pp. 273-300. School of American Research Press, Santa Fe.

Hesse, B. 1982. Animal domestication and oscillating climates. Journal of Ethnobiology 2:1-15.

Hidalgo, J. 1985. Ecological complementarity and tribute in Atacama: 1683-1792. En Andean Ecology and Civilization, editado por S. Masuda, I. Shimada y C. Morris, pp. 161-184. Tokio Press, Tokio.
Holden, T. 1991. Evidence of prehistoric diet from northern Chile: Coprolites, gut contents and flotation samples from Tulan Quebrada. World Archaeology 22:320-331.

Jackson, D. y A. Benavente 2010. Complejización de los cazadores y recolectores en Chiu Chiu, río Loa Medio (Norte de Chile). Estudios Atacameños 39:5-20.

Kelly, R.L. 1992. Mobility/Sedentism: Concepts, archaeological measures, and effects. Annual Review of Anthropology 21:43-66.

Knudson, K. 2008. Tiwanaku Influence in the South Central Andes: Strontium Isotope Analysis and Middle Horizon Migration. Latin American Antiquity 19:3-23.

Knudson, K. y C. Torres-Rouff 2009. Investigating cultural heterogeneity in San Pedro de Atacama, northern Chile, through biogeochemistry and bioarchaeology. American Journal of Physical Anthropology 138:473-485.

Korstanje, A. 2005. La Organización del Trabajo en Torno a la Producción de Alimentos en Sociedades Agro-Pastoriles Formativas (Provincia de Catamarca, Argentina). Tesis para optar al grado de doctor en Arqueología, Facultad de Ciencias Naturales e Instituto Miguel Lillo, Universidad Nacional de Tucumán, Tucumán.

Kuznar, L. 2001. Risk sensitivity and value among Andean pastoralists: Measures, models, and empirical tests. Current Anthropology 42:432-440.

Lema, V.S., C. Della Negra y V. Bernal 2012. Explotación de recursos vegetales silvestres y domesticados en Neuquén: implicancias del hallazgo de restos de maíz y algarrobo en artefactos de molienda del Holoceno Tardío. Magallania 40:229-247.

Liu, L., W. Ge, S. Bestel, D. Jones, J. Shi, Y. Song y X. Chen 2011. Plant exploitation of the last foragers at Shizitan in the Middle Yellow River Valley China: evidence from grinding stones. Journal of Archaeological Science 38:3524-3532.

Llagostera, A., A. Barón y L. Bravo 1984. Investigaciones arqueológicas en Tulor 1. Estudios Atacameños 7:105-115.

Logan, A., C. Hastorf y D. Pearsall 2012. "Let's drink together": early ceremonial use of maize in the Titicaca basin. Latin American Antiquity 23:235-258.

Martínez, J.L. 1998. Pueblos del Chañar y el Algarrobo: Los Atacamas en el Siglo XVII. DIBAM, Santiago.

Mauldin, R. 1993. The relationship between ground stone and agricultural intensification in Western New Mexico. Kiva 58:317-330.

McClure, S., M.A. Jochim y M.C. Barton 2006. Human behavioral ecology, domestic animals, and land use during the transition to agriculture in Valencia, Eastern Spain. En Human Behavioral Ecology and the Transition to Agriculture, editado por D. Kennett y B. Winterhalder, pp. 197-216. University of California Press, Berkeley.

McRostie, V. 2007. La Transición Arcaico-Formativa en la Quebrada de Tulán, Sur del Salar de Atacama, Chile. Evidencias Arqueobotánicas. Tesis para optar al grado de Antropólogo con mención en Arqueología. Departamento de Antropología, Universidad de Chile, Santiago. 
----2013. The Role of Plant Production in Subsistence and Cultural Changes During the Formative Period in the Atacama Puna, Southern Andes, Chile (1400 B.C.- A.D. 500). A ReEvaluation Based on the Analyses of Microfossils Attached to Hoes and Grinding Tools, and Isotopic Analyses of Human Bones. Doctoral dissertation, Institute of Archaeology, University College London, London.

Messerli, B., M. Grosjean, G. Bonani, A. Burgi, M.A. Geyh, K. Graf, K. Ramseyer, H. Romero, U. Schotterer, H. Schreier y M. Vuille 1993. Climate change and natural resource dynamics of the Atacama Altiplano during the last 18,000 years; a preliminary synthesis. Mountain Research and Development 13:117-127

Messner, T.C. 2008. Woodland Period People and Plant Interactions: New Insights from Starch Grain Analysis. Doctoral dissertation, Temple University, PA. UMI Microform, Ann Arbor.

Minnis, P. 1992. Earliest plant cultivation in the desert borderlands of North America. En The Origins of Agriculture: An International Perspective, editado por C.W. Cowan y P.J. Watson, pp. 121-141. Smithsonian Press, Washington.

Nester, P.L., E.M. Gayó, C. Latorre, T.E. Jordan y N. Blanco 2007. Perennial stream discharge in the hyperarid Atacama Desert of northern Chile during the latest Pleistocene. Proceedings of the National Academy of Sciences 104:19724-19729.

Núñez, L. 1986-1987. Análisis multidisciplinario de domesticación y crianza inicial de camélidos en Los Andes del Norte de Chile. Manuscrito inédito en posesión del autor.

----1992. Ocupación arcaica en la Puna de Atacama: secuencia, movilidad y cambio. En Prehistoria Sudamericana: Nuevas Perspectivas, editado por B. Meggers, pp. 85-108. Taraxacum, Washington, DC.

----1995. Evolución de la ocupación y organización del espacio Atacameño. Aspectos dinámicos. En Agua, Ocupación del Espacio y Economía Campesina en la Región Atacameña, editado por P. Pourrut y L. Núñez, pp. 18-60. Universidad Católica del Norte - ORSTOM, Antofagasta.

----2005. La naturaleza de la expansión aldeana durante el Formativo Tardío en la Cuenca de Atacama. Chungara Revista de Antropología Chilena 37:165-193.

Núñez, L., C. Agüero, B. Cases y P. de Souza 2003. El campamento minero Chuquicamata-2 y la explotación cuprífera prehispánica en el Desierto de Atacama. Estudios Atacameños 25:7-34.

Núñez, L., I. Cartajena, C. Carrasco, P. de Souza y M. Grosjean 2006. Emergencia de comunidades pastoralistas Formativas en el Sureste de la Puna de Atacama. Estudios Atacameños 32:93-117.

Núñez, L. y T. Dillehay 1979. Movilidad Giratoria, Armonía y Desarrollo en los Andes Meridionales; Patrones de Tráfico e Interacción Económica. Dirección General de Investigaciones Tecnológicas, Universidad del Norte, Antofagasta.

Núñez, L., V. McRostie e I. Cartajena 2009. Consideraciones sobre la recolección vegetal y la horticultura durante el Formativo temprano en el Sureste de la Cuenca de Atacama. Darwiniana 47:56-75.

Núñez, L. y C. Santoro 1988. Cazadores de la Puna Seca y Salada del área Centro Sur Andina (Norte de Chile). Estudios Atacameños 9:13-65.
----2011. El tránsito Arcaico-Formativo en la Circumpuna y Valles Occidentales del Centro Sur Andino: Hacia los cambios "Neolíticos". Chungara Revista de Antropología Chilena 43:487-530

Olivera, D. 1998. Cazadores y pastores tempranos de la Puna Argentina. Past and Present in Andean Prehistory and Early History Vol. 42, pp.153-180. Etnografiska Museet i Göteborg, Göteborg.

Olivera, D. y H. Yacobaccio 1999. Estudios de paleodieta en poblaciones humanas de los Andes del Sur a través de isótopos estables. V Congreso Nacional de Paleopatología, pp. 1-11. Alcalá La Real, Jaén.

Palacios, R. y M. Brizuela 2005. Prosopis: historia y elementos para su domesticación. Agrociencia 9:41-51.

Pearsall, D. 1989. Adaptation of prehistoric hunter-gatherers to the high Andes: The changing role of plant resources. En Foraging and Farming: The Evolution of Plant Exploitation, editado por D.R. Harris y G.C. Hillman, pp. 318-332. UnwinHyman, London.

----2007. The impact of maize on subsistence systems in South America: an example from the Jama river valley, coastal Ecuador (1999). En The Emergence of Agriculture: A Global View, editado por T. Denham y P. White, pp. 83-103. Routledge, New York.

Pérez, S. 2010.Variabilidad en la producción de palas y/o azadas líticas de la Puna Argentina. Estudios Atacameños 40:5-22.

Pollard, G.C. 1970. The Cultural Ecology of Ceramic Stage Settlement in the Atacama Desert. Doctoral dissertation, Department of Anthropology, Columbia University, New York.

----1971. Cultural change and adaptation in the central Atacama desert of Northern Chile. Nawpa Pacha 9:41-68.

Price, D.T. y A.B. Gebauer 1995. New Perspectives on the transition to agriculture. En Last Hunters, First Farmers: New Perspectives on the Prehistoric Transition to Agriculture, editado por D.T. Price y A.B. Gebauer, pp. 3-20. School of American Research Press, Santa Fe.

Rech, J., J. Quade y J. Betancourt 2002. Late Quaternary paleohydrology of the central Atacama Desert (lat 22-24 ${ }^{\circ}$ S), Chile. Geological Society of America Bulletin 114:334-348.

Rindos, D. 1984. The Origins of Agriculture: An Evolutionary Perspective. Academic Press, Orlando.

Rivera, M. 2005. Arqueología del Desierto de Atacama: La Etapa Formativa en el Área de Ramaditas/Guatacondo. Universidad Bolivariana, Santiago.

Rodríguez, R., O. Matthei y M. Quezada 1983. Flora Arbórea de Chile. Editorial de la Universidad de Concepción, Concepción.

Shimada, M. e I. Shimada 1985. Prehistoric Llama breeding and herding on the North Coast of Peru. American Antiquity 50:3-26.

Smith, B. 2001. Low-level food production. Journal of Archaeological Research 9:1-43.

----2006. Documenting domesticated plants in the archaeological record. En Documenting Domestication. New Genetic and Archaeological Paradigms, editado por M.A. Zeder, D. Bradley, E. Emshwiller y B.D. Smith, pp. 15-24. University of California Press, California. 
Stahl, A.B. 1989. Plant food processing: implications for dietary quality. En Foraging and Farming: The Evolution of Plant Exploitation, editado por D.R. Harris y G.C. Hillman, pp. 171196. Unwin-Hyman, London.

Staller, J. 2006. La domesticación de paisajes: ¿Cuáles son los componentes primarios del Formativo? Estudios Atacameños $32: 43-57$

Staller, J. y R. Thompson 2000. Reconsiderando la introducción del maíz en el Occidente de América del Sur. Bulletin de l'Institut Francais d'Études Andines 30:123-156.

Tamblay, J.A. 1990. Aspectos del Proceso de Formación del Sitio Arqueológico. Tesis para optar al grado de Antropológo con mención en Arqueología, Departamento de Antropología, Universidad de Chile, Santiago.

Tarragó, M. 1989. Contribución al Conocimiento Arqueológico de Las Poblaciones de Los Oasis de San Pedro de Atacama en Relación con los Otros Pueblos Puneños, en Especial el Sector Septentrional del Valle Calchaqui. Tesis para optar al grado de Doctor en Arqueología, Facultad de Humanidades y Artes, Universidad Nacional de Rosario.

Terrell, J.E., J.P. Hart, S. Barut, N. Cellinese, A. Curet, T. Denham, C. Kusimba, K. Latinis, R. Oka, J. Palka, M.E. Pohl, K.O. Pope, P.R. Williams, H. Haines y J.E. Staller 2003. Domesticated landscapes: The subsistence ecology of plant and animal domestication. Journal of Archaeological Method and Theory 10:323-368.

Thomas, C., A. Benavente, I. Cartajena y G. Serracino 1995. Topater, un cementerio temprano: una aproximación simbólica. Hombre y Desierto 9:159-170.

Tykot, R., F. Falabella, M.T. Planella, E. Aspillaga, L. Sanhueza y C. Becker 2009. Stable isotopes and archaeology in central Chile: methodological insights and interpretative problems for dietary reconstruction. International Journal of Osteoarchaeology 19:156-170.

Uribe, M. y C. Carrasco 1999. Tiestos y piedras talladas de Caspana: la producción alfarera y lítica en el Período Tardío en el Loa Superior. Estudios Atacameños 18:55-71.
Valero-Garcés, B., M. Grosjean, A. Schwalb, M.A. Geyh, B. Messerli y K. Kelts 1996. Limnology of Laguna Miscanti; evidence for mid to late Holocene moisture changes in the Atacama Altiplano (Northern Chile). Journal of Paleolimnology 16(1):1-21.

Vidal, A. 2007. Patrones de Uso de los Recursos Vegetales Durante el Periodo Formativo (1000aC-500dC) en San Pedro de Atacama: Oasis y Quebradas. Tesis para optar al grado de Antropológo con mención en Arqueología, Departamento de Antropología, Universidad de Chile, Santiago.

Wheeler, J. 1995. Evolution and present situation of the South American Camelidae. Biological Journal of the Linnean Society 54:271-195.

Whitehead, W.T. 2006. Redefining plant use at the Formative site of Chiripa in the southern Titicaca Basin. En Andean Archaeology III: North and South, editado por W. Isbell y H. Silverman, pp. 258-278. Springer, New York.

Winterhalder, B. y D. Kennet 2006. Behavioral ecology and the transition from hunting and gathering to agriculture. En Behavioral Ecology and the Transition to Agriculture, editado por D. Kennet y B. Winterhalder, pp. 1-21. University of California Press, London.

Wollstonecroft, M., P. Ellis, G.C. Hillman y D. Fuller 2008. Advances in plant food processing in the Near Eastern Epipalaeolithic and implications for improved edibility and nutrient bioaccessibility: an experimental assessment of Bolboschoenus maritimus (L.) Palla (sea club-rush). Vegetation History and Archaeobotany 17:19-27.

Wright, H.E. 1993. Environmental determinism in Near Eastern Prehistory. Current Anthropology 34:458-469.

Wright, K. 1993. Early Holocene ground stone assemblages in the Levant. Levant 25:93-111.

----1994. Ground-Stone tools and hunter-gatherer subsistence in Southwest Asia: Implications for the transition to farming. American Antiquity 59:238-263.

\section{Notas}

* Este manuscrito está basado en la tesis doctoral de la autora (McRostie 2013).

1 Esta categoría se refiere a microfósiles con baja resolución taxonómica.
2 Así como también lo requiere a futuro la otra especie arbórea de estos oasis Geoffroea decorticans. 
